\title{
Albrecht Kessler
}

\section{Anthropogene Änderungen des Strahlungshaushalts der Erdoberfläche}

Zur Problematik der Rekonstruktion vorzeitlicher Strahlungsklimate in Mitteleuropa 
ANTHROPOGENE ÄNDERUNGEN DES STRAHLUNGSHAUSHALTS DER ERDOBERFLÄCHE Zur Problematik der Rekonstruktion vorzeitlicher Strahlungsklimate in Mitteleuropa ${ }^{122}$

Mit 2 Abbildungen und 1 Tabelle

Albrecht KesSLER

Summary: The influence of man on the radiation budget of the earth's surface in the past

Problems are discussed which result from the reconstruction of the radiation budget of the earth's surface of postglacial times in Middle Europe. Data of measured net radiation components of a grass surface and of a pine stand are presented. The importance of cloud amount on the budget components is referred to.

\section{Einleitung}

Die Strahlungsbilanz, über deren anthropogene Veränderungen hier berichtet wird, ist die wichtigste Komponente des Wärmeumsatzes an der Erdoberfläche. Sie ist die zentrale steuernde Größe des Klimas der bodennahen Luftschicht.

Die Gleichung für die Strahlungsbilanz Q einer horizontalen Fläche hat folgende Form, wenn die Strahlungsströme aus dem oberen Halbraum positiv und die von unten nach oben gerichteten Ströme negativ gezählt werden:

$\mathrm{Q}=\mathrm{Q}_{\mathrm{K}}+\mathrm{Q}_{\mathrm{L}}=(\mathrm{S}+\mathrm{H}-\mathrm{R})+(\mathrm{A}-\mathrm{E})$, mit $\mathrm{G}=\mathrm{S}+\mathrm{H}, \mathrm{R}=(\mathrm{S}+\mathrm{H}) \alpha_{\mathrm{K}}, \mathrm{E}=\mathrm{A} \alpha_{\mathrm{L}}+\varepsilon \sigma \mathrm{T}^{4}$,

$\mathrm{Q}$ Gesamtstrahlungsbilanz; $\mathrm{Q}_{\mathrm{K}}$ bzw. $\mathrm{Q}_{\mathrm{L}}$ kurzwellige und langwellige Bilanzen - die Grenze zwischen kurzwelligem solaren und langwelligem terrestrischen, die atmosphärische Gegenstrahlung und die Emission der Erdoberfläche umfassenden Spektralbereich liegt bei $4 \mu \mathrm{m}$; $S$ direkte Sonnenstrahlung; $\mathrm{H}$ gestreute Sonnenstrahlung = Himmelsstrahlung; G kurzwellige Globalstrahlung; R kurzwellige Reflexstrahlung; $a_{\mathrm{K}}=\mathrm{R} / \mathrm{G}$ kurzwellige Albedo; A langwellige Gegenstrahlung der Atmosphäre einschließlich Wolken; E langwellige Strahlungsemission der Fläche einschließlich reflektierter, langwelliger Gegenstrahlung, deren Betrag allerdings gering ist; $\alpha_{\mathrm{L}}$ langwellige Albedo; $\varepsilon$ Emissionsvermögen der langwellig ausstrahlenden Oberfläche; $\sigma$ Stefan-Boltzmann-Konstante; T Temperatur der Oberfläche.

\section{Strablungsbilanz und Vorzeitklima}

Um die Auswirkungen anthropogener Veränderungen der Strahlungsbilanz auf die allgemeine, postglaziale Klimaentwicklung abschätzen zu können, muß jeweils der dem Eingriff vorausgehende Urzustand rekonstruierbar sein.

Hierfür bieten sich im wesentlichen zwei Methoden an. Man kann einmal aktualistisch vorgehen und die rezenten Unterschiede zwischen natürlichen und anthropogenen Oberflächentypen bestimmen und dann die Ergebnisse auf frühere Zustände zu übertragen versuchen. Zum anderen kommt eine mehr theoretische Behandlung durch Aufstellung von Klimamodellen in Frage. Die Anwendung von Klimamodellen setzt allerdings ebenfalls umfangreiche Messungen verschiedener Parameter und ihrer Variabilitätseigenschaften voraus.

Bevor wir auf die anthropogenen Veränderungen im Postglazial zu sprechen kommen, sollte auf einen Umstand besonders aufmerksam gemacht werden. Durch die allgemeine postglaziale Klimaentwicklung veränderte sich die Vegetation allmählich. Für Mitteleuropa sind folgende Hauptphasen zu nennen: Baumlose Tundra, Birken-Kiefernwälder, Eichenmischwald, Buchenzeit (FIrBAs 1949, 1952). Theorie und bisherige Erfahrungen zeigen, daß bei gleichen äußeren Einflüssen durch verschiedene Oberflächentypen der Wärme- und Wasserhaushalt der bodennahen Luft unterschiedlich gesteuert wird mit allen Konsequenzen für das jeweilige Bestandsklima bzw. allgemein für das Mesoklima, so daß man feststellen kann, daß in den einzelnen Phasen unterschiedlicher Oberflächentypen im Postglazial die Abwandlung des „äußeren Klima“ durch die Unterlage sehr verschieden gewesen ist. Statt des „äußeren Klima“ könnte man auch formulieren: der advektiven Komponente des Großklimas.

Wenn wir uns hier auf die Behandlung von anthropogenen Veränderungen beschränken wollen, so sollte man sich jedoch darüber im klaren sein, daß das Problem der Klimaänderung oder -beeinflussung durch Veränderungen der Erdoberfläche auch für die Zeitabschnitte gilt, in denen der menschliche Einfluß als unbedeutend angesehen werden kann, beziehungsweise daß die natürlichen Veränderungen der Erdoberfläche einen gleich starken, wie auch immer gerichteten Einfluß auf das Klima gehabt haben können wie die anthropogenen Eingriffe.

Die erste wichtige Phase menschlichen Eingriffs in den Landschaftshaushalt setzte im Neolithikum ein (W AHLE 1980). Das Waldareal wurde zu Gunsten freier Flächen zurückgedrängt. Weitere solche Abschnitte besonderer Aktivität mit Vergrößerung der Acker- und Wiesenflächen sind die Rodungsperioden der neueren Zeit. Hier wäre die frühmittelalterliche Rodung der Mittelgebirge zu nennen.

\footnotetext{
1) In memoriam Prof. Dr. ERNST WahLE (1889-1981), der mich vor 30 Jahren mit Problemen der prähistorischen Landschaft vertraut machte.

2) Überarbeitete Fassung eines Vortrages, der auf dem Symposium über Klimageschichte der letzten beiden Interglaziale (Leitung Prof. Dr. Dr. h. c. B. Frenzel) 1980 an der Akademie der Wissenschaften und der Literatur in Mainz gehalten wurde.
} 
Schließlich kommt seit dem 19. Jahrhundert durch Verstädterung, Straßenbau, Kanalisation und Industrialisierung ein weiterer Typ der Oberflächenveränderung hinzu. Er beschränkt sich nicht nur auf die Veränderung der Strahlungsverhältnisse. Durch Beschleunigung des Abflusses von den versiegelten oder dränierten Oberflächen wird der Wasserhaushalt und indirekt über den latenten Wärmestrom auch der Energiehaushalt der Erdoberfläche maßgeblich verändert. Außerdem tritt zunehmend stärker ein künstlicher Energieinput in die untere Atmosphäre durch Heizung und industrielle Immissionen auf.

Der Rekonstruktion der vorzeitlichen Strahlungs- und Wärmeumsätze an der Erdoberfläche stehen zur Zeit noch erhebliche Hindernisse im Wege.

Folgende Umstände sind zu nennen:

a) Die Strahlung an der Erdoberfläche wird entscheidend von den Bewölkungsverhältnissen beeinflußt. Geeignete Informationen über die Bewölkung im Verlauf des Postglazials gibt es nicht. Sie werden nur schwer zu erhalten sein. Auch von Klimasimulationen mit Hilfe von Zirkulationsmodellen sind keine verläßlichen Werte für die einzelnen Phasen in Mitteleuropa zu erwarten.

Abb. 1 zeigt die Abhängigkeit der Strahlungsströme vom Wolken-Bedeckungsgrad. Die Untersuchungen wurden in der südlichen Oberrheinebene bei Hartheim durchgeführt. Große Unterschiede in der Abhängigkeit vom Bewölkungsgrad sind zwischen Winter- und Sommermonaten festzustellen. Im Winter nimmt beispielsweise die Gegenstrahlung A mit zunehmender Bewöl-

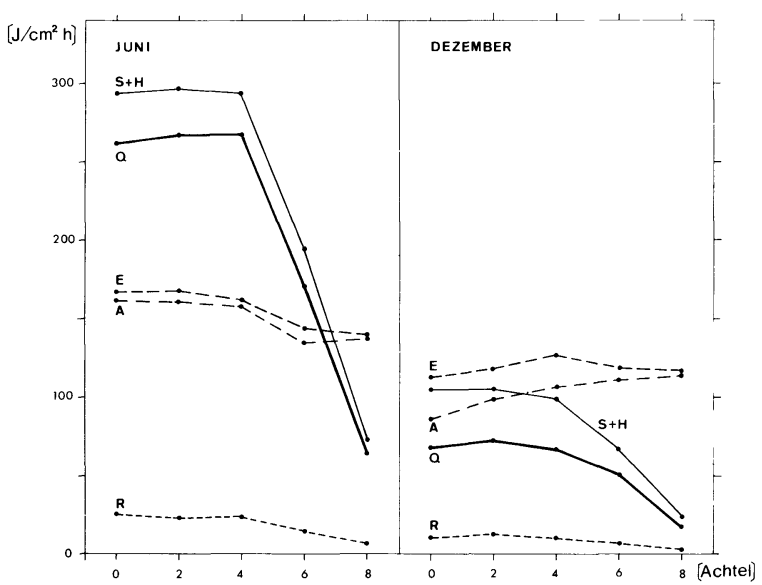

Abb. 1: Abhängigkeit der Strahlungsbilanz $\mathrm{Q}$, der Globalstrahlung $\mathrm{S}+\mathrm{H}$, der Reflexstrahlung $\mathrm{R}$, der Gegenstrahlung $\mathrm{A}$ und der langwelligen Ausstrahlung E vom Wolkenbedeckungsgrad in Achteln; mittlere Stundensummen für die Zeit des höchsten täglichen Sonnenstandes (12-13 h MEZ) für einen Kiefernwald in Hartheim in der südlichen Oberrheinebene, in Joule/( $\left.\mathrm{cm}^{2} \mathrm{~h}\right)$; Mittelwerte der Jahre 1974-1977

Dependence of net radiation $Q$, global radiation $S+H$, reflected global radiation $\mathrm{R}$, atmospheric longwave radiation $\mathrm{A}$ and terrestrial longwave radiation $\mathrm{E}$ on the cloud amount (eights); mean hourly sums, 12-13 h MET, for a pine stand kung zu, im Sommer dagegen ab. Diese unerwartete Abnahme im Sommer ist damit zu erklären, daß die stärker bedeckten Tage im allgemeinen die kühleren sind, so daß die Wirkung erhöhter Himmelsbedeckung, die bei gleicher Atmosphärentemperatur und Dampfdruck zur Vergrößerung der Gegenstrahlung führen müßte, durch ein niedrigeres Temperatur- und Dampfdruckniveau an diesen Tagen überkompensiert wird.

Globalstrahlung $\mathrm{S}+\mathrm{H}$ und Strahlungsbilanz $\mathrm{Q}$ zeigen im Bereich $0 / 8$ bis $4 / 8$ Himmelsbedeckung geringe Veränderungen. Bei höheren Bewölkungsgraden, wie sie in Mitteleuropa am häufigsten sind und wohl auch in der Vorzeit waren, ist eine starke Reduktion bei $\mathrm{S}+\mathrm{H}$ und $\mathrm{Q}$ festzustellen. So führt eine Fehlannahme von $1 / 8$ des Bedeckungsgrades in diesem Bereich zu einer Fehlschätzung bei der Globalstrahlung und der Strahlungsbilanz von $25-50 \%$ !

Diese wenigen Hinweise zur Problematik der Himmelsbedeckung bei der Rekonstruktion früherer Strahlungsverhältnisse mögen hier genügen. Weitere Informationen über die Änderung der Strahlungsverhältnisse einer Rasenoberfläche in Abhängigkeit vom Bedeckungsgrad findet man bei KASTEN und CZEPLAK (1980). Im Zusammenhang mit dem rezenten Kohlendioxidanstieg der Atmosphäre und der Abschätzung der zu erwartenden Temperaturzunahme hat kürzlich HuNT (1981) auf ähnliche Schwierigkeiten hingewiesen, die durch Variation der Himmelsbedeckung eintreten.

Weitere Schwierigkeiten bei der Rekonstruktion bereiten folgende Gesichtspunkte:

b) Der Bodenwasserhaushalt steuert über die Albedo direkt und über den Wärmehaushalt indirekt die Strahlungsvorgänge an der Erdoberfläche. Deshalb sind mehr und qualitativ bessere Informationen über das Bodenwasser früherer Epochen notwendig als bisher vorliegen. Paläoökologen müßten sich dieser Aufgabe verstärkt annehmen.

c) Die bisher vorliegenden Untersuchungen zum Strahlungs- und Wärmehaushaltklima von rezenten Standorten und Oberflächentypen in Mitteleuropa sind noch verhältnismäßig spärlich. Einen Vergleich der Strahlungsverhältnisse verschiedener Oberflächentypen findet man bei KESSLER (1985b). Bedauerlicherweise liegen auch kaum längere Beobachtungsreihen vor. Damit fehlen Informationen über die natürlichen Variabilitätseigenschaften der Vorgänge in Abhängigkeit von den diversen Klimaelementen und ihren Schwankungen. Dadurch wird die Anwendung der Ergebnisse auf frühere Zustände sehr erschwert.

Greifen wir nur ein Beispiel heraus. Selbst bei der kurzwelligen Oberflächenalbedo $\alpha_{\mathrm{K}}$, also einem zentral wichtigen Parameter, sind noch viele Fragen offen, vor allem, wenn man bedenkt, daß verschiedene Oberflächentypen auf gleichartige Einstrahlungsänderungen sehr unterschiedlich reagieren können. In diesem Zusammenhang ist zu erinnern etwa an die Abhängigkeit der Albedo vom Bewölkungsgrad, von der Bodenfeuchte, vom Azimut des Sonnenstandes, von der Struktur und von der Art des Bestandsaufbaus verschiedener Vegetationsdecken etc. 
Als weiteres Problem ist zu sehen, daß Albedowerte der wichtigsten im Postglazial verbreiteten Oberflächentypen gar nicht hinreichend bekannt sind. Eine erste systematische Bearbeitung über die Abhängigkeit der Albedo von den erwähnten Parametern am Beispiel eines jungen Kiefernwaldes wurde von KESSLER (1985a) vorgelegt.

Eine charakteristische und in ihrem Einfluß nachhaltige Veränderung früherer Epochen wurde durch die Rodungstätigkeit herbeigeführt. Diese lief in Mitteleuropa in mehreren Phasen ab. Der Einfluß auf Mikro- und Mesoklima muß - abgesehen von der gravierenden Oberflächentypenveränderung selbst - deshalb erheblich gewesen sein, weil durch diese Maßnahmen große Areale betroffen wurden. Im folgenden wird am Beispiel mehrjähriger Dauermessungen von allen Strahlungskomponenten über einem Kiefernwald (Hartheim 47 $56^{\prime} \mathrm{N}, 7^{\circ} 37^{\prime} \mathrm{E}, 201 \mathrm{~m} \mathrm{NN}$ ) und über einer Rasenoberfläche (Hamburg $53^{\circ} 38^{\prime} \mathrm{N}, 10^{\circ} 00^{\prime} \mathrm{E}, 14 \mathrm{~m} \mathrm{NN}$ ) gezeigt, mit welchen Auswirkungen der Rodungstätigkeit auf die Strahlungsflüsse bei entsprechender Änderung des Oberflächentyps gerechnet werden muß. Der Einfluß auf die Strahlungsbilanz $Q$ ist im wesentlichen auf Albedoänderungen $\left(a_{K}\right)$ und Veränderungen der Temperatur $(T)$ der langwellige Strahlung emittierenden Oberfläche zurückzuführen. Letzteres ist für die verschiedenen Bestandstypen nur sehr schwer mit Modellen anzugeben, zumal zur Überprüfung der Simulationen die Beobachtungsgrundlagen weitgehend fehlen. Die Oberflächentemperatur ist unter anderem eine Funktion der verschiedenen an der Erdoberfläche ablaufenden Energieumsätze. Außer der Strahlungsbilanz $Q$ sind daran der Bodenwärmestrom $B$ und die turbulenten Ströme fühlbarer und latenter Wärme ( $\mathrm{L}$ und V) beteiligt. Weitere Angaben über die beiden Strahlungsmeßstellen findet man bei GARTHE et al. (1985), JAEGER (1978), JAEger und Kessler (1980), Kessler et al. (1979), SCHOtT (1980).

\section{DieKomponenten der Strablungsbilanz übereinem Kiefern wald und über einer Rasenoberfläche in Mitteleuropa}

Die allgemeine klimatische Situation beider Standorte kann kurz folgendermaßen charakterisiert werden. Hamburg (Rasenoberfläche) liegt im maritimen Bereich der reliefarmen Norddeutschen Tiefebene. Die mittlere Jahrestemperatur ist mit $9,5^{\circ} \mathrm{C} \mathrm{um} 1^{\circ} \mathrm{C}$ niedriger als in Hartheim (Kiefernwald), desgleichen die mittlere Jahresschwankung der Temperatur (hierbei handelt es sich wohlgemerkt jeweils um die Wetterhüttentemperatur, die nicht mit der Bestandsoberflächentemperatur identisch ist). Die Jahresniederschlagsmenge von Hamburg (1931-60, $714 \mathrm{~mm}$ ) ist ca. $100 \mathrm{~mm}$ höher als in Hartheim. Die Hamburger Station besaß im Meßzeitraum (1974-1977) in den Monaten Oktober bis Februar und im Juli höhere Bedeckungsgrade. Im März war es umgekehrt und die übrigen Monate zeigten bei beiden Standorten etwa gleiche Bedeckungsverhältnisse. Das Hartheimer Klima ist als trockenwarm und kontinental
Tabelle 1: Durchschnittliche Jabresmittel der Tagessummen der Strah lungsbilanz $Q$ und ibrer Komponenten einer Rasenoberfläche in Hamburg und eines Kiefernwaldes in Hartheim, Mittelwerte der Jabre 1974-1977 in Joule/(cm² Tag)

Average yearly mean values of daily sums of net radiation $Q$ and its components (Joule $/ \mathrm{cm}^{2}$ day) of a grass surface and a pine stand

\begin{tabular}{lcc}
\hline & $\begin{array}{c}\text { Kiefernbestand } \\
\text { bei Hartheim }\end{array}$ & $\begin{array}{c}\text { Rasen bei } \\
\text { Hamburg }\end{array}$ \\
\hline Strahlungsbilanz $\mathrm{Q}_{=} \mathrm{Q}_{\mathrm{K}}+\mathrm{Q}_{\mathrm{L}}$ & 752 & 484 \\
Kurzwellige Bilanz $\mathrm{Q}_{\mathrm{K}}=\mathrm{S}+\mathrm{H}-\mathrm{R}$ & 978 & 780 \\
Langwellige Bilanz $\mathrm{Q}_{\mathrm{L}}=\mathrm{A}-\mathrm{E}$ & -226 & -296 \\
Globalstrahlung S + H & 1082 & 981 \\
Reflexstrahlung R & 104 & 201 \\
Gegenstrahlung A & 2871 & 2897 \\
Langwellige Ausstrahlung E & 3097 & 3193 \\
Albedo $a$ in \% & 10,1 & 21,6 \\
\hline
\end{tabular}

getönt $\mathrm{zu}$ bezeichnen. Die Gebirgszüge im Westen und Osten der Oberrheinebene bewirken häufig Föhneffekte mit Auflockerungen der Schichtbewölkung, was vor allem in den Wintermonaten zu den Bewölkungs- und Strahlungsunterschieden zwischen Hamburg und Hartheim führt.

Tab. 1 enthält die durchschnittlichen Jahresmittelwerte der Strahlungsflüsse. Danach ist die Strablungsbilanz Q des Rasens bei Hamburg um 36\% niedriger als $Q$ des Kiefern. waldes bei Hartheim. Die Unterschiede sind im wesentlichen zurückzuführen:

1) auf eine um den Betrag von $101 \mathrm{~J} /\left(\mathrm{cm}^{2} \mathrm{Tag}\right)$ höhere Globalstrahlung $\mathrm{G}$ in Hartheim, bedingt durch die Bewölkungsunterschiede und den niedrigeren Sonnenstand in Hamburg. Hierbei handelt es sich demnach um Einflüsse des Groß- und Mesoklimas und der geographischen Lage;

2) auf einen in der gleichen Größenordnung $\left(97 \mathrm{~J} /\left(\mathrm{cm}^{2}\right.\right.$ Tag)) liegenden geringeren Betrag der Reflexstrahlung in Hartheim. Hier kommt der Unterschied der kurzwelligen Albedo $a_{\mathrm{K}}$ zum Tragen. Der Hartheimer Kiefernwald (Albedo-Jahresmittel 10,1\%) hat eine etwa halb so große Albedo als der Rasen bei Hamburg (Albedo 21,6\%);

3) auf einen wiederum etwa gleich hohen Differenzbetrag $\left(96 \mathrm{~J} /\left(\mathrm{cm}^{2} \mathrm{Tag}\right)\right)$, um den die langwellige Strahlungsemission $\mathrm{E}$ des Kiefernwaldes niedriger ist. Es ist besonders bemerkenswert, daß der Rasen bei Hamburg trotz eines erheblich geringeren kurzwelligen Strahlungsgenusses $(\mathrm{S}+\mathrm{H}-\mathrm{R})$ eine höhere Oberflächentemperatur und damit ein höheres $E$ besitzt. Dieser Umstand ist u.a. auf die unterschiedliche Struktur der beiden Oberflächen zurückzuführen. Beim Kiefernwald handelt es sich im Gegensatz zum Rasen um eine tiefgestaffelte Strahlungsumsatzschicht, deren höhere Rauhigkeit auch die turbulenten Wärmeströme intensiviert. Wie die Abb. $2 \mathrm{a}$ und $2 \mathrm{~b}$ zeigen, gelten diese Aussagen nicht nur im Jahresmittel sondern für jeden einzelnen Monat des Jahres. In diesen Differenzen äußert sich die unterschiedliche Steuerung des gesamten Wärmehaushalts durch die beiden Oberflächentypen. 

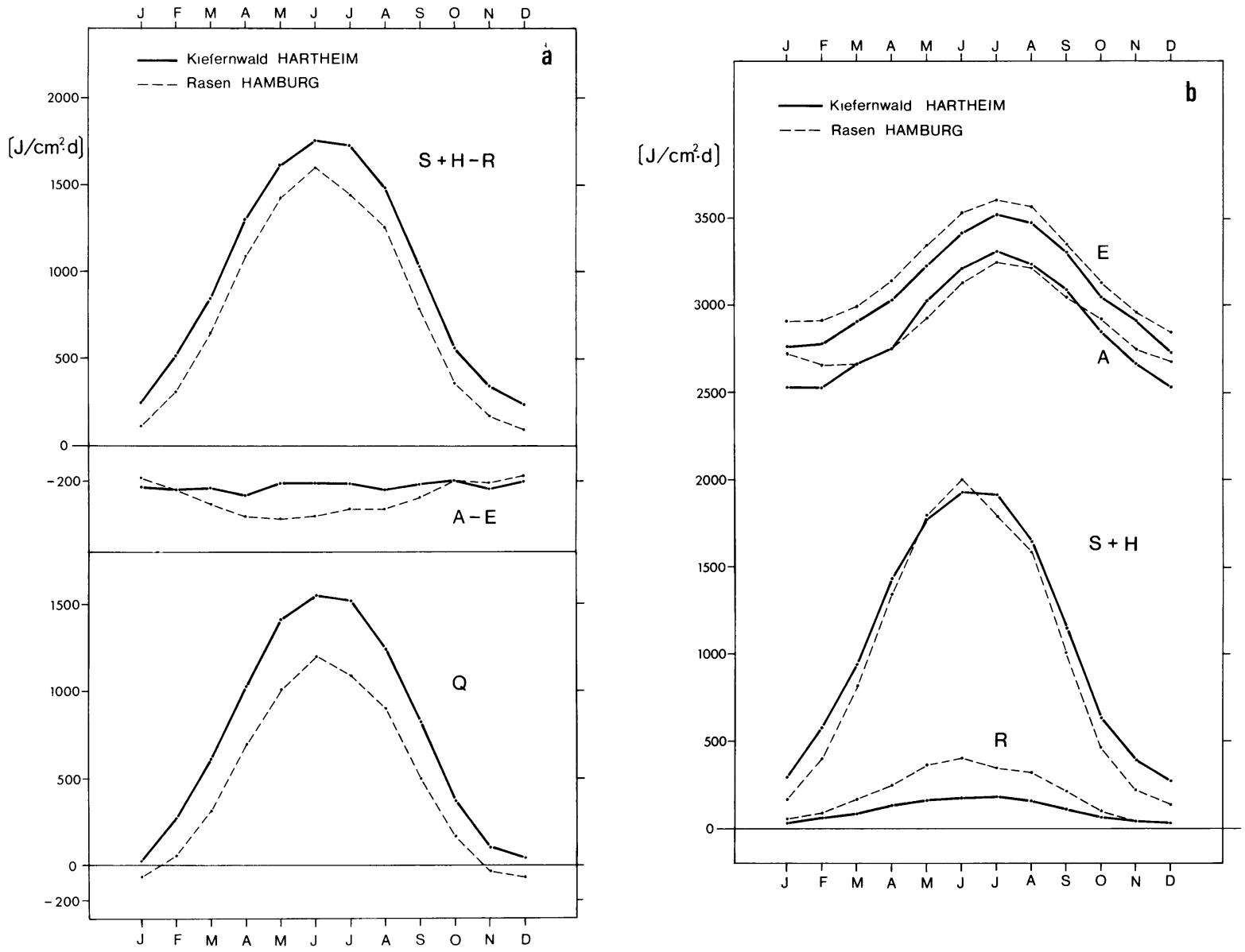

Abb. 2a: Jahresgang der mittleren Tagessummen in Joule $/\left(\mathrm{cm}^{2} \mathrm{Tag}\right)$ der kurzwelligen Strahlungsbilanz $\mathrm{Q}_{\mathrm{K}}=\mathrm{S}+\mathrm{H}-\mathrm{R}$, der langwelligen Strahlungsbilanz $Q_{L}=A-E$ und der Gesamtstrahlungsbilanz $Q=Q_{K}+Q_{L}$; Mittelwerte der Jahre 1974-1977, jeweils für eine Rasenoberfläche bei Hamburg und einen Kiefernwald bei Hartheim

Annual variation of the mean daily sums (Joule $/ \mathrm{cm}^{2}$ day) of net shortwave radiation $\mathrm{Q}_{\mathrm{K}}$, net longwave radiation $\mathrm{Q}_{\mathrm{L}}$ and total net radiation Q

Abb. 2b: Jahresgang der mittleren Tagessummen in Joule/( $\left.\mathrm{cm}^{2} \mathrm{Tag}\right)$ der Globalstrahlung $\mathrm{S}+\mathrm{H}$, der Reflexstrahlung $\mathrm{R}$, der langwelligen Ausstrahlung E und der Gegenstrahlung A; Mittelwerte der Jahre 1974-1977, jeweils für eine Rasenoberfläche bei Hamburg und einen Kiefernwald bei Hartheim

Annual variation of the mean daily sums (Joule/ $\mathrm{cm}^{2}$ day) of global radiation $S+H$, reflected global radiation $R$, terrestrial radiation $E$ and atmospheric longwave radiation $\mathrm{A}$

\section{Zusammenfassung und Danksagung}

Am Beispiel von Strahlungsmessungen über einem Kiefernwald wurde auf die Bedeutung des Bedeckungsgrades für die kurzwelligen und die langwelligen Strahlungsflüsse hingewiesen. Daraus geht hervor, daß zur Rekonstruktion des Wärme- und Strahlungshaushaltes postglazialer Ökotope, seien sie nun natürlicher oder mehr anthropogener Natur, und zur Abschätzung ihres Anteils an den postglazialen Klimaänderungen die Bewölkungsverhältnisse der entsprechenden Epochen möglichst genau bekannt sein müssen.
Auf Grund von 4jährigen Strahlungsmessungen über einer Rasenoberfläche und einem ca. $5 \mathrm{~m}$ hohen Kiefernwald ergab sich, daß die Strahlungsbilanz $Q$ des Rasens um ca. 1/3 niedriger als $Q$ des Kiefernwaldes war. Zum größeren Teil ist die Differenz auf Unterschiede in der Albedo und der Oberflächentemperatur zurückzuführen. Die Größenordnung beider Effekte ist etwa gleich.

$\mathrm{Da}$ diese Unterschiede oberflächenspezifisch sind und streng genommen nur für Rasen und Kiefernwald gelten, lassen sie sich nur zur Abschätzung einer derartigen Oberflächenveränderung anwenden. Für andere Typen der an- 
thropogenen Oberflächenveränderung im Postglazial sollten geeignete Messungen durchgeführt werden, um eine verläßliche Datengrundlage zu schaffen, die mit anderen Methoden nur schwerlich zu erhalten ist.

Der vorliegende Beitrag wurde dankenswerterweise durch die Deutsche Forschungsgemeinschaft und durch das Klimaprogramm der Bundesregierung (BMFT) gefördert.

\section{Literatur}

Deutscher Wetterdienst:Das Klimader Bundesrepublik Deutschland. Lieferung 1, Offenbach 1979.

Firbas, F.: Waldgeschichte Mitteleuropas. Jena, Bd. 1, 1949; Bd. 2 , 1952.

GarThe, H. J., Jaeger, L. u. Kessler, A.: Langzeitmessungen zum Strahlungs- und Energiehaushalt eines Kiefernwaldes. Tagungsber. Nationalpark Bayer. Wald 5, 1985.

Hunt, B. G.: An examination of some feedback mechanisms in the carbon dioxide climate problem. In: Tellus 33, 1981, 78-88.
JAEGER, L.: Die klimatologische Meßstation/Hartheim des Meteorologischen Instituts der Universität Freiburg im Breisgau. In: Ber. Naturf. Ges. Freiburg i. Br. 68, 1978, 47-73.

JAEGER, L. u. KesSLER, A.: Langzeitmessungen der Strahlungsbilanz und ihrer Komponenten über einem Kiefernstand der südlichen Oberrheinebene. In: Allg. Forst- u. Jagdztg. 151, 1980, 210-218.

KASTEN, F. u. CzEPLAK, G.: Solar and terrestrial radiation dependent on the amount and type of cloud. In: Solar Energy 24, 1980, 177-189.

KESSLER, A.: Über die kurzwellige Albedo eines Kiefernwaldes. Eine klimatologische Langzeitstudie. In: Meteorol. Rdsch. 38, 1985 a, 82-91.

- : Heat balance climatology. World Survey of Climatology Vol. 1A. Amsterdam, London, New York, Tokyo 1985b.

Kessler, A., JAEger, L. u. Schott, R.: Die Auswirkungen der Sonnenfinsternis vom 29. April 1976 auf die Energieströme an der Erdoberfläche. In: Meteorol. Rdsch. 32, 1979, 109-115.

Reichsamt für Wetterdienst: Klimakunde des Deutschen Reiches, Bd. 2 Tabellen. Berlin, 1939.

Sснотт, R.: Untersuchungen über die Energiehaushaltskomponenten in der atmosphärischen Grenzschicht am Beispiel eines Kiefernbestandes in der Oberrheinebene (Hartheim/Rh.). Ber. Deutscher Wetterd. 153, 1980.

WAHLE, E.: Ur- und Frühgeschichte im mitteleuropäischen Raum. 5. Aufl., München 1980. 